\title{
Author Correction: A missense mutation in the HECT domain of NEDD4L identified in a girl with periventricular nodular heterotopia, polymicrogyria, and cleft palate
}

\author{
Koji Kato ${ }^{1,2} \cdot$ Fuyuki Miya ${ }^{3,4} \cdot$ Ikumi Hori $^{1} \cdot$ Daisuke leda $^{1} \cdot$ Kei Ohashi $^{1} \cdot$ Yutaka Negishi $^{1} \cdot$ Ayako Hattori $^{1} \cdot$ \\ Nobuhiko Okamoto ${ }^{5}$ Mitsuhiro Kato ${ }^{6} \cdot$ Tatsuhiko Tsunoda $^{3,4} \cdot$ Mami Yamasaki $^{7} \cdot$ Yonehiro Kanemura $^{8,9}$. \\ Kenjiro Kosaki ${ }^{10} \cdot$ Shinji Saitoh $\mathbb{1}^{1}$
}

Published online: 26 April 2019

(c) The Author(s), under exclusive licence to The Japan Society of Human Genetics 2019

\section{Correction to: Journal of Human Genetics \\ https://doi.org/10.1038/jhg.2017.53 \\ published online 18 May 2017}

Since the publication of this article, it has been brought to our attention, that the identified mutation (NM_015277: c. $2617 \mathrm{G}>\mathrm{A}$; p.Glu873Lys) is identical with the mutation (NM_001144967: c.2677 G > A; p.Glu893Lys) reported by Broix et al (Nature Genetics 48, 1349-1358, 2016 https:// doi.org/10.1038/ng.3676). Therefore the mutation is not novel but recurrent. Accordingly, the word "novel" should be deleted throughout the article including the title. Thus, the title should read "A missense mutation in the HECT domain of NEDD4L identified in a girl with periventricular nodular heterotopia, polymicrogyria, and cleft palate."

It was also brought to our attention, that there is an error in Fig. 1d. In the original Fig. 1d, the amino acid numbers of the four variants in the HECT domain were incorrect. The amino acid numbers were determined using NM_001144967. The new amino acid numbers have been determined using NM_015277. The previous variants read p.Tyr679Cys, p.Gln694His, p.Glu893Lys and p. Arg897Gln. The variants should now read p.Tyr659Cys, p. Gln674His, p.Glu873Lys and p.Arg877Gln. The correct Figure is shown below. The authors apologize for any inconvenience caused.
These authors contributed equally: Koji Kato, Fuyuki Miya.

The original article can be found online at https://doi.org/10.1038/jhg. 2017.53 .

Shinji Saitoh

ss11@med.nagoya-cu.ac.jp

1 Department of Pediatrics and Neonatology, Nagoya City University Graduate School of Medical Sciences, Nagoya, Japan

2 Department of Pediatrics, Nagoya University Graduate School of Medicine, Nagoya, Japan

3 Department of Medical Science Mathematics, Medical Research Institute, Tokyo Medical and Dental University, Tokyo, Japan

4 Laboratory for Medical Science Mathematics, RIKEN Center for Integrative Medical Sciences, Yokohama, Japan

5 Department of Medical Genetics, Osaka Medical Center and Research Institute for Maternal and Child Health, Osaka, Japan
6 Department of Pediatrics, Showa University School of Medicine, Tokyo, Japan

7 Department of Neurosurgery, Takatsuki General Hospital, Osaka, Japan

8 Division of Regenerative Medicine, Institute for Clinical Research, Osaka National Hospital, National Hospital Organization, Osaka, Japan

9 Department of Neurosurgery, Osaka National Hospital, National Hospital Organization, Osaka, Japan

10 Center for Medical Genetics, Keio University School of Medicine, Tokyo, Japan 
Fig. 1 Patient's brain magnetic resonance imaging (MRI), photograph and the identified NEDD $4 L$ mutation. Brain MRI was acquired at 8 months of age. Her parents gave written consent for publication of the photograph. (a) Axial slice of T1-weighted image showing bilateral periventricular nodular heterotopia (arrow head) and polymicrogyria (arrow). (b) Photograph of the patient taken at 3 years of age showing distinctive facies. (c) Sanger sequencing of the NEDD4L mutation. The patient has a heterozygous c. $2617 \mathrm{G}>\mathrm{A}$ mutation (arrow) not present in the parents. (d) Previously published mutations in the HECT domain of NEDD4L associated with periventricular nodular heterotopia (red arrows, below) and our patient's

mutation (blue arrow, above). A full color version of this figure is available at the Journal of Human Genetics journal online
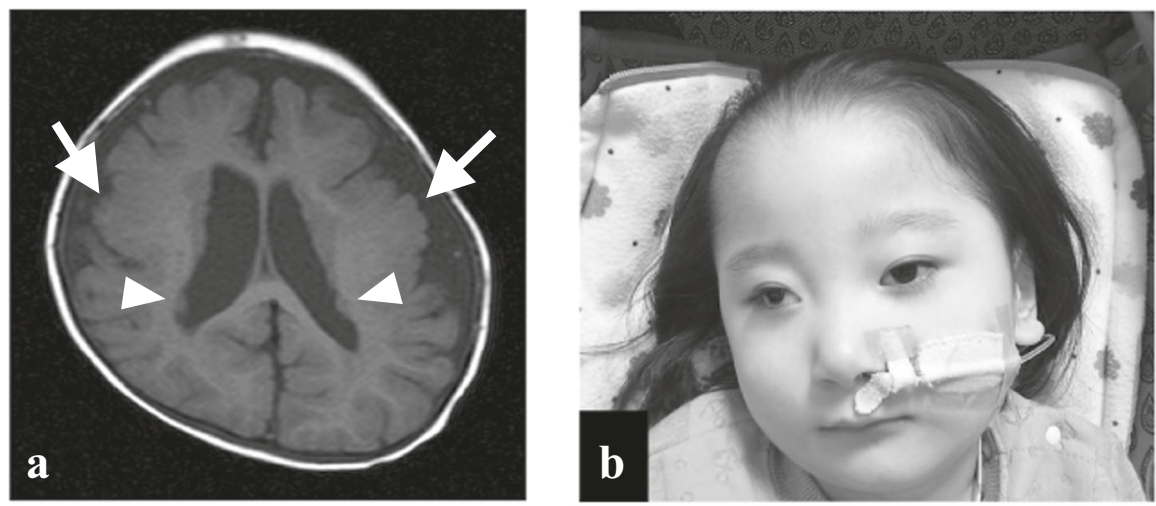

c

$$
\text { Patient }
$$

Father

Mother

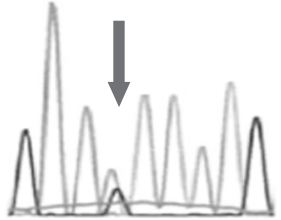

Normal GCCGAAAAG Mutant GCCAAAAAG

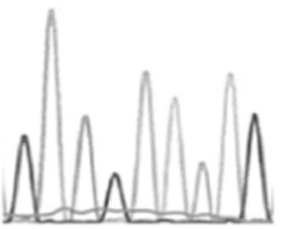

GCCGAAAAG

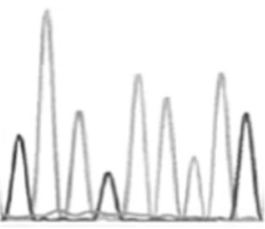

GCCGAAAAG

d

p.Glu873Lys

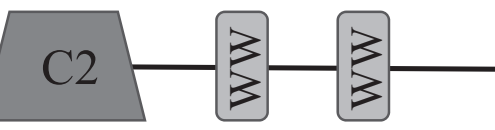

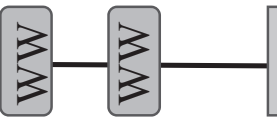

p.Tyr659Cys

p.Arg877Gln

p.Gln674His

p.Glu873Lys 